Vol. 10(11), pp. 246-257, November 2016

DOI: 10.5897/AJPS2016.1448

Article Number: B2983BA61529

ISSN 1996-0824

Copyright (C) 2016

African Journal of Plant Science

Author(s) retain the copyright of this article

http://www.academicjournals.org/AJPS

\title{
Genetic diversity in lowland, midaltitude and highland African maize landraces by morphological trait evaluation
}

\author{
Stephen Asare ${ }^{1}$, Antonia Y. Tetteh ${ }^{2 \star}$, Patrick Twumasi ${ }^{1}$, Kingsley B. Adade ${ }^{2}$ and \\ Richard A. Akromah ${ }^{1}$ \\ ${ }^{1}$ Department of Crop and Soil Science, Kwame Nkrumah University of Science and Technology Kumasi, Ghana. \\ ${ }^{2}$ Department of Biochemistry and Biotechnology, Kwame Nkrumah University of Science and Technology \\ Kumasi, Ghana.
}

Received 28 July, 2016; Accepted 29 September, 2016

\begin{abstract}
Genetic diversity information is a resource for improvement in crop productivity and trait performance, however, there is scanty information on genetic diversity estimates in the maize landraces covering the major geographical regions of Africa. In the current study, the genetic diversity of 35 landraces originating from lowland, midaltitude and highland regions of Africa and held in the IITA Genetic Resource Center, Ibadan, Nigeria, were evaluated using morphological trait evaluation. The landraces were tested in non-stressed environments in Ghana by evaluation of 27 traits. A large within and between genetic variability was identified which increased from highland to lowland populations and was highest in the midaltitude population. Genetic similarity coefficients ranged from 0.00 to 0.80 with mean of $0.26 \pm 0.18$ across the three populations, and $0.23 \pm 0.16,0.29 \pm 0.18$, and $0.38 \pm 0.25$ in the midaltitude, lowland and highland populations, respectively. A total of 21 discriminant traits were identified from the principal components analysis. A UPGMA cluster analysis and PCA biplot produced four main clusters which provide a sound basis for exploitation of heterosis. Nine distant landraces were identified majority of which produced grain yield exceeding $5.0 \mathrm{Mg} \mathrm{ha}^{-1}$. In terms of improvement in grain yield, earliness and drought tolerance, TZm-14, TZm-41, TZm-242, TZm-37, TZm-1360, TZm1376, TZm-1367, TZm-4, and TZm-270 would be useful. A large genetic diversity resides in the African maize landraces which could be conserved and exploited for maize improvement.
\end{abstract}

Key words: Maize, genetic diversity, African maize landraces, morphological traits, cluster analysis, PCA.

INTRODUCTION

Maize (Zea mays L.) is the most widely grown cereal crop in Sub-Saharan Africa (SSA) covering 29 million hectares or $15.7 \%$ of the arable land area with 1.7 million hectares in highland areas, 8.0 million hectares in the mid-altitude

*Corresponding author. E-mail: atetteh.sci@knust.edu.gh, aytetteh@gmail.com. Tel: +233-20-113-4416. Fax: +233-322060137.

Author(s) agree that this article remains permanently open access under the terms of the Creative Commons Attribution License 4.0 International License 
regions, and 12.3 million hectares in the tropical lowlands (FAOSTAT, 2013), all in smallholder systems except in South Africa. Demand for maize has increased to meet food requirements for population growth, as feed for livestock and poultry and for biofuel ethanol production. At a population growth rate of over $2 \%$ per annum, SSA needs to double her maize production to feed an estimated population of 1.5 billion by year 2050 (FAO, 2006). Compared to global increase in maize production of $2.2 \%$ per annum at annual yield growth of $1.5 \mathrm{Mg} \mathrm{ha}^{-1}$, maize yield in SSA has barely increased by little over $1 \%$ in 50 years reaching an average yield of $1.8 \mathrm{Mg} \mathrm{ha}^{-1}$ in 2011 , about a quarter of the world average yield of 5.5 $\mathrm{Mg} \mathrm{ha}^{-1}$ (Fischer et al., 2014).

Constraints to maize yield increase in Africa, include low soil fertility (Gibbon et al., 2007), limited availability and low adoption of modern improved varieties which achieve farm yield of only $25 \%$ of the potential yield (FAOSTAT, 2013), lack of irrigation facilities and low agricultural input. Other limiting factors include limited labor, and uncertainties with crop success currently arising from climate change effects (Cairns et al., 2013; IPCC, 2007) which drive farmers to have preference for their landraces.

The landraces, being historic and dynamic genotypes, and having evolved from natural and anthropogenic selection system (Bellon and van Etten, 2013) exhibit some buffering effect to climate change effects including extreme heat stress and drought (Mercer et al., 2012; Mercer and Perales, 2010). By their wide genetic base, requirement for low agronomic input, better storage properties, desirable culinary characteristics, and some genotypes possessing superior agronomic characteristics than the improved cultivars (Amanor, 2013; Bellon, 2004), the landraces represent unique class of germplasm for exploitation and development.

Currently, only few attempts have been made to examine the genetic diversity in the SSA landraces. The need to evaluate the entire collection to estimate the breadth of genetic diversity and identify genotypes with important traits such as high grain yield and climate adaptive traits is of prime importance.

Both morphological and molecular methods are employed in estimating genetic diversity in germplasm collections. Although morphological evaluation is limited by effect of environment on trait expression, exhibits low heritability, is time consuming, labour intensive, requires a large population size, and does not cover the genome (Botha and Venter, 2000; Smith and Smith, 1992), it offers an unparalleled means of identification of phenotypic variation.

Reports on genetic diversity estimation among maize collections of North America (James et al., 2002; Smith, 1986; Goodman and Stuber, 1983; Kahler et al., 1983), CIMMYT (Warburton et al., 2005, 2002; Xia et al., 2005; Carvalho et al., 2002), European maize (Hartings et al., 2008; Okumus, 2007; Rebourg et al., 2001), and Asia populations (Enoki et al., 2002; Yuan et al., 2000) abound, whereas a parallel study in African maize is emerging. The few reports of maize genetic variation in Africa include those of localized zones of Ethiopia (Legesse et al., 2007; Beyene et al., 2006), Ghana (Oppong et al., 2014; Obeng-Antwi, 2007, Obeng-Antwi et al., 2012) and Malawi, Zambia, and Zimbabwe in southern Africa (Magorokosho, 2006). Assessment of genetic diversity covering a wide geographical area is required not only to identify and quantify the variation, but also to explore the basis of variation in the African maize germplasm.

The research carried out by Sanou et al. (1997), Beyene et al. (2006), Legesse et al. (2007) and Magorokosho (2006) considered isozyme variability in West African maize landraces, molecular characterization of Ethiopian genotypes, and combination of morphological and molecular characterization of southern Africa genotypes, respectively. Morphological evaluation constitutes an appropriate tool for identifying useful genotypes, grouping germplasm, and identifying relationships among the groups. Breeders rely on morphological characterization as the first step in distinguishing genotypes, removing obvious duplicates, and acquiring a guide to selection of parents.

In the context of maize cultivation, six mega environments are defined in Africa, on the basis of climate, elevation and soil type into the highlands with elevation above 1600 m.a.s.l., upper humid, lower humid, and dry midaltitude elevation of 900 to 1600 m.a.s.l, and humid and dry lowlands below 800 m.a.s.l. It is believed that the landraces that have had long exposure and survival in these conditions have differentiated in their respective environments hence worth examining for their genetic diversity estimates.

The International Institute of Tropical Agriculture (IITA) has in store over 1,000 maize landraces collected from many countries in Africa. The objective of this research was to determine the genetic diversity in maize originating from three environments namely, lowland, midaltitude, and highland regions of SSA using phenotypic characterization. The information will be useful for identifying genotypes for broadening the genetic base in the gene pools of maize improvement programs and provide a guide for conservation and management of maize.

\section{MATERIALS AND METHODS}

\section{Plant material}

Thirty-five lowland, midaltitude and highland landraces originating from 12 countries in SSA were sampled from the IITA maize collection (Table 1). An open-pollinated lowland genotype, 'Obatanpa GH' was included as a check. Accessions were planted in the wet season in two consecutive years, March to July 2011 and April to August 2012 in the Agricultural Experiment Station of the Kwame Nkrumah University of Science and Technology, Kumasi, Ghana, in randomized complete block design with three 
Table 1. The landraces of lowland, midaltitude and highland origins in SSA sampled from the IITA maize collection.

\begin{tabular}{|c|c|c|c|c|c|c|c|}
\hline $\mathrm{S} / \mathrm{N}$ & Acc. name & Local name & Origin & $\begin{array}{l}\text { Mega- } \\
\text { environment }\end{array}$ & $\begin{array}{c}\text { Altitude } \\
\text { (m.a.s.I.) }\end{array}$ & $\begin{array}{l}\text { Longitude } \\
\text { (degrees) }\end{array}$ & $\begin{array}{c}\text { Latitude } \\
\text { (degrees) }\end{array}$ \\
\hline 1 & $\mathrm{TZm}-2$ & Mziava & Tanzania & Lowland & 310 & 38.30 & -6.02 \\
\hline 2 & $\mathrm{TZm}-4$ & Katumani & Tanzania & Midaltitude & 1000 & 37.57 & -3.38 \\
\hline 3 & $\mathrm{TZm}-5$ & Katumani & Tanzania & Midaltitude & 1000 & 37.55 & -3.33 \\
\hline 4 & $\mathrm{TZm}-8$ & Katumani & Tanzania & Midaltitude & 1240 & 32.72 & -2.62 \\
\hline 5 & TZm-13 & Katumani & Tanzania & Midaltitude & 1160 & 31.02 & -3.30 \\
\hline 6 & TZm-14 & Katumani & Tanzania & Midaltitude & 1160 & 30.10 & -4.65 \\
\hline 7 & TZm-20 & Katumani & Tanzania & Highland & 2100 & 31.77 & -8.35 \\
\hline 8 & TZm-33 & Walingombe & Tanzania & Midaltitude & 1480 & 34.58 & -8.85 \\
\hline 9 & TZm-37 & Walingombe & Tanzania & Midaltitude & 1500 & 34.90 & -8.73 \\
\hline 10 & TZm-41 & Walingombe & Tanzania & Midaltitude & 1520 & 35.92 & -7.73 \\
\hline 11 & $\mathrm{TZm}-42$ & Walingombe & Tanzania & Midaltitude & 1580 & 36.08 & -7.70 \\
\hline 12 & TZm-242 & Chintubulungu & Zambia & Midaltitude & $>900$ & 31.50 & -9.75 \\
\hline 13 & $\mathrm{TZm}-251$ & Solweizi & Zambia & Midaltitude & $>900$ & 26.00 & -12.5 \\
\hline 14 & $\mathrm{TZm}-270$ & Makandakunda & Zambia & Midaltitude & $>900$ & 23.00 & -13.5 \\
\hline 15 & $\mathrm{TZm}-273$ & Chinyinji & Zambia & Midaltitude & $>900$ & 22.75 & -13.25 \\
\hline 16 & TZm-275 & Mwinhunga & Zambia & Midaltitude & $>900$ & 24.33 & -12.25 \\
\hline 17 & TZm-301 & Karal & Chad & Lowland & 300 & 14.80 & 12.92 \\
\hline 18 & TZm-384 & Oyo bokouele & Congo & Lowland & 290 & 16.10 & -1.07 \\
\hline 19 & TZm-385 & Oyo bokouele & Congo & Lowland & 315 & 16.30 & -1.00 \\
\hline 20 & $\mathrm{TZm}-1084$ & Zunde ngabu & Malawi & Lowland & 100 & 31.50 & -16.47 \\
\hline 21 & TZm-1095 & Balaka & Malawi & Lowland & 100 & 34.97 & -16.57 \\
\hline 22 & $\mathrm{TZm}-1356$ & Guinea local & Equatorial Guinea & Midaltitude & $600-1220$ & 1.50 & 10.00 \\
\hline 23 & $\mathrm{TZm}-1358$ & Guinea local & Equatorial Guinea & Midaltitude & $600-1220$ & 1.50 & 10.00 \\
\hline 24 & $\mathrm{TZm}-1359$ & Chebolosinik & Equatorial Guinea & Midaltitude & $600-1221$ & 1.50 & 10 \\
\hline 25 & $T Z m-1360$ & Githigu & Kenya & Highland & 2057 & $N A^{1}$ & NA \\
\hline 26 & $\mathrm{TZm}-1367$ & Mahindi & Kenya & Highland & & NA & NA \\
\hline 27 & $\mathrm{TZm}-1376$ & Kiragoli & Kenya & Highland & $>1600$ & NA & NA \\
\hline 28 & $\mathrm{TZm}-1413$ & Magadishu to Baidoba & Somalia & Lowland & 497 & 44.97 & 2.47 \\
\hline 29 & $\mathrm{TZm}-1424$ & Makambako Iringa & Tanzania & Highland & 1900 & 34.90 & -8.73 \\
\hline 30 & $\mathrm{TZm}-1434$ & NA & Togo & Lowland & $<500$ & NA & NA \\
\hline 31 & TZm-1437 & NA & Togo & Lowland & $<500$ & NA & NA \\
\hline 32 & $\mathrm{TZm}-1514$ & Chintubulungu & Zambia & Midaltitude & 1235 & 31.50 & -9.75 \\
\hline 33 & $\mathrm{TZm}-1516$ & Kanjilimini & Zambia & Midaltitude & 1235 & 28.83 & -9.75 \\
\hline 34 & $\mathrm{TZm}-1521$ & Mali 2 & Guinea & Midaltitude & $600-1500$ & NA & NA \\
\hline 35 & $\mathrm{TZm}-1523$ & Tangue & Guinea & Lowland & 188 & NA & NA \\
\hline 36 & 'Obatanpa GH' & NA & Ghana & Lowland & 277 & -1.67 & 6.67 \\
\hline
\end{tabular}

replications. The planting density was 42,000 plants/ha in $6 \mathrm{~m} \times 0.6$ $\mathrm{m}$ rows having 15 plants per row. The experimental site was located at longitude $1.61^{\circ} \mathrm{W}$ and latitude $6.6^{\circ} \mathrm{N}$ and elevation of $277 \mathrm{~m}$.a.s.l. Fertilizer application was equivalent to $120: 60: 40 \mathrm{~kg} \mathrm{ha}^{-1}$ of $\mathrm{N}-\mathrm{P}_{2} \mathrm{O}_{5-}$ $\mathrm{K}_{2} \mathrm{O}$ plus $50 \mathrm{~kg} \mathrm{ha}^{-1}$ sulphate of ammonia applied at 21 days after planting and at ear emergence. Irrigation was carried out as and when needed. Maize stem borers (Busseola fusca, Sesamia calamistis) and cutworms (Agrotis spp.) were controlled using Conpyrifos $48 \%$ (1.0 to $1.5 \mathrm{~L} \mathrm{ha}^{-1}$ ) and Cymethoate Super (1.0 to $\left.1.5 \mathrm{~L} \mathrm{ha}^{-1}\right)$.

\section{Morpho-phenological trait evaluation}

Morpho-phenological traits were evaluated by employing the
IBPGRI and CIMMYT (1991) maize descriptor list. Five qualitative traits, silk color (pale yellow $=1$; red $=2$ ), cob colour $(0=$ red; 5=white), kernel arrangement (1=regular; 2=irregular; $3=$ straight; and $4=$ spiral), kernel texture ( $1=$ flint; $3=$ mixed; $5=$ dent), and principal grain colour ( $0=$ white; $1=$ other colors) were measured with appropriate instruments. A total of 22 morpho-phenological traits on ten competitive plants per plot comprising anthesis date (AD), silking date (SD), anthesis-silking interval (ASI), tassel length (TL), ear leaf length (ELL), ear leaf width (ELW), plant height (PHT), ear height (EHT), stalk diameter (StD), stay green (SG), ear length (EL), cob diameter (CD), number of rows per ear (NRE), and number of kernels per row (NKR) were measured. After harvest, the number of ears per plant $(E N)$, kernel length $(K L)$, kernel width (KW), kernel thickness (KT), ear weight (EWT), and one-hundredkernel weight $(\mathrm{HKW})$ expressed as mass of 100 kernels adjusted to 
$15 \%$ moisture content were measured. Grain yield was calculated as shelled grain weight per plot adjusted to $125 \mathrm{~g} / \mathrm{kg}$ moisture content

\section{Data analyses}

Data were analyzed by analysis of variance using the General Linear Model of SAS 9.3 (SAS Institute, 2011). Pairwise genetic distances based on Pearson correlation coefficient were computed on the standardized data matrix. Mean genetic distance of the entire population was calculated. The hierarchical Unweighted Pair Group Method with Arithmetic Averages (UPGMA) clustering of the distance matrix was carried out to generate a dendrogram. Statistical significance of the dendrogram was determined by bootstrap analysis (Felsenstein, 1985) using the PAST software (Hammer et al., 2001). Finally, a principal components analysis (PCA) and biplots determined the discriminatory power of the traits and revealed relationships among traits and accessions. The NTSYS pc 2.2 software (Rohlf, 2009) was employed for all multivariate analysis.

\section{RESULTS}

\section{Within and between population differences}

Ear characteristics included $57 \%$ pale yellow silks, $92 \%$ white cobs and $62 \%$ dent kernels in predominantly regular kernel arrangement. Mean values, minimum and maximum, standard deviation, mean squares and coefficient of variation of the 22 phenotypic traits are presented in Table 2. The populations exhibited a large within population differentiation except for anthesis-silking interval and a large between population variation for all traits but tassel length, ear leaf width, plant height, and stalk diameter. A trend of increasing variability based on the number of significant mean squares was identified in 16 traits in highland, 20 traits in lowland, and 21 in midaltitude populations (Table 2).

Low to moderate correlation coefficients were found between traits. Anthesis-silking interval showed predominantly low nonsignificant negative correlations with all other traits whereas ear leaf width showed moderate significant positive correlations with all traits. Similarly, grain yield showed low to moderate significant correlation with all traits except anthesis date, silking date, ear leaf length, ear length, and kernel thickness (Table 3).

On earliness, pollen shed and silking required a mean of $58.62 \pm 5.67$ and $62.68 \pm 6.10$ days, respectively, hence protandry of $4.08 \pm 1.66$ days. This was similar to the check, 'Obatanpa GH', which showed mean number of days to anthesis and silking of 48.8 and 52.5 days, respectively, and protandry of $3.7 \pm 1.11$ days. Anthesissilking interval ranged from 2.33 to 6.17 days. Important accessions that were less protandrous than the check were lowland TZm-1523 (3.2 days) and TZm-301 (3.3 days), midaltitude TZm-37 (3.2 days), TZm-1516 (3.5 days), and TZm-1521 (3.3 days), and highland TZm1367(3.20 days) and TZm-1376 (2.3 days).

Variations in traits on the basis of accession means are provided. Plant height varied from 139.95 to $207.95 \mathrm{~cm}$ and ear height from 55.00 to $112.98 \mathrm{~cm}$. Tassel length of 42.69 to $53.41 \mathrm{~cm}$, stalk diameter of 15.38 to $22.97 \mathrm{~mm}$ and stay green from 33.33 to $96.25 \%$. The accessions showed average ear leaf length and ear leaf width of 79.16 and $8.50 \mathrm{~cm}$, respectively, compared to the check with 84.16 and $9.86 \mathrm{~cm}$, respectively. The largest ear leaf width of $11.11 \mathrm{~cm}$ was exhibited by TZm-270. All yield component traits had lower values than the improved check. Ear length ranged from 13.40 to $18.07 \mathrm{~cm}$, ear diameter 32.48 to $46.49 \mathrm{~mm}$, cob diameter 20.94 to 39.00 $\mathrm{mm}$, number of rows per ear 9.60 to 16.92 , number of kernels per row 21.33 to 36.85 , and ear number ranged from 1.00 to 1.27. On individual plant basis, $T Z m-1434$, $\mathrm{TZm}-1367$, TZm-384, and TZm-1413 produced ears having 20 to 22 rows per ear, a slight increase above the common value of 12 to 20 . Similarly, a high number of kernels per row of 48 to 54 was identified in TZm-273, TZm-301 TZm-1084, TZm-1359, and TZm-1360 exceeding the recorded 36 to 44 range for improved genotypes. Accessions with at least two ears per plant included TZm-1367, TZm-2, TZm-384, TZm-1434, TZm1437, TZm-33, TZm-1359, TZm-385, TZm-41, and TZm1356. The hundred kernel weight ranged from 46.88 to $93.76 \mathrm{~g}$. Overall mean grain yield on plant basis was $4.48 \pm 2.56 \mathrm{Mg} \mathrm{ha}^{-1}$ with a range of 0.7 to $12.5 \mathrm{Mg} \mathrm{ha}^{-1}$ and accession mean grain yield of 1.73 to $6.20 \mathrm{Mg} \mathrm{ha}^{-1}$ compared to a check yield of $6.29 \mathrm{Mg} \mathrm{ha}^{-1}$. Kernel characteristics were highly variable.

\section{Genetic distance}

Genetic similarity represented by squared Pearson correlation coefficients ranged from 0.0 to 0.82 with a mean of $0.26 \pm 0.18$ (Table 4) for the entire data. Ten accession pairs including the check were most similar with similarity coefficients of 0.82 (Obatanpa/TZm-1413), 0.80 (Obatanpa/TZm-1521; TZm-251/TZm-37), 0.78 (TZm-1521/TZm-1413), 0.76 (TZm-20/TZm-1367), 0.73 (TZm-385/TZm-1521), 0.72 (TZm-384/TZm-1376), 0.71 (TZm-384/TZm-1367; TZm-1424/TZm-1434), and 0.70 (TZm-1523/TZm-1413; TZm-2/TZm-1424). The highland accessions were separated by genetic distance of $0.31 \pm 0.24$ in a range of 0.03 to 0.76 , midaltitude accessions by mean $0.25 \pm 0.16$, in a range of 0.00 to 0.80 , and lowland accessions by $0.27 \pm 0.20$ covering a range of 0.00 to 0.82 .

Nine accession pairs having genetic distances of 0.0 were most dissimilar. These included TZm-1095/TZm-41 of lowland Malawi/Midaltitude Tanzania, TZm-1084/TZm384 of lowland Malawi/ lowland Congo, TZm-1095/TZm1434, TZm-1360/TZm-1434 of highland Kenya/lowland Togo, TZm-1376/TZm-2 of highland Kenya/lowland Tanzania, TZm-1437/TZm-1521 of lowland Togo/Midaltitude Guinea, TZm-1523/TZm-2 of lowland Guinea/ lowland Tanzania,TZm-2/TZm-20 lowland Tanzania/highland Tanzania, and TZm-37/TZm-42 both 


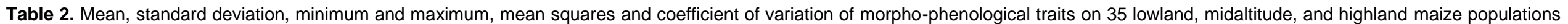
including a check evaluated in Kumasi in 2011 and 2012 major rainy seasons.

\begin{tabular}{|c|c|c|c|c|c|}
\hline \multirow{2}{*}{ No. } & \multirow{2}{*}{ Trait } & \multicolumn{3}{|c|}{ Population } & \multirow{2}{*}{$\begin{array}{l}\text { Overall mean } \\
\text { (Mean square) }\end{array}$} \\
\hline & & Highland & Midaltitude & $\begin{array}{l}\text { Lowland } \\
\end{array}$ & \\
\hline 1 & AD (days) & $59.79 \pm 3.36^{a}(52-66) 15.73^{*} 5.62$ & $58.52 \pm 5.77^{\mathrm{b}}(43-73) 114.98^{* \star *}, 9.86$ & $58.21 \pm 6.23^{\mathrm{b}}(45-71) 174.43^{* * *}, 10.69$ & $58.62 \pm 5.67\left(267.42^{* * *}\right)$ \\
\hline 2 & SD (days) & $63.27 \pm 3.74^{\mathrm{a}}(55-71) 32.52^{* *} 5.91$ & $62.77 \pm 6.3^{\mathrm{ab}}(46-78) 133.74^{* \star *}, 10.03$ & $62.27 \pm 6.5^{\mathrm{b}}(48-77) 196.14^{\star * *}, 10.54$ & $62.68 \pm 6.10\left(114.09^{\star}\right)$ \\
\hline 3 & ASI (days) & $3.48 \pm 1.58^{\mathrm{b}}(2-9) 3.61,45.29$ & $4.25 \pm 1.74^{\mathrm{a}}(2-12) 3.65,40.91$ & $4.05 \pm 1.46^{\mathrm{a}}(2-8) 3.48,36.00$ & $4.08 \pm 1.66\left(7.57^{\star \star \star}\right)$ \\
\hline 4 & $\mathrm{TL}(\mathrm{cm})$ & $46.62 \pm 6.51(30.5-63.5) 6.22,13.96$ & $47.05 \pm 7.17(23-74) 39.56^{\star * *}, 15.25$ & $47.56 \pm 7.97(11.5-69) 50.71^{* * *}, 16.75$ & $47.14 \pm 7.34(106.09)$ \\
\hline 5 & $\mathrm{ELL}(\mathrm{cm})$ & $78.77 \pm 15.95^{\mathrm{b}}(16-105) 59.52,20.25$ & $78.18 \pm 17.49^{b}(21.6-113) 314.71^{* \star *}, 22.38$ & $81.12 \pm 15.71^{\mathrm{a}}(26-112) 316.51^{\star \star \star}, 19.35$ & $79.16 \pm 16.80\left(1870.09^{\star \star}\right)$ \\
\hline 6 & $\mathrm{ELW}(\mathrm{cm})$ & $8.52 \pm 1.59(4-12.6) 2.40^{*}, 18.62$ & $8.44 \pm 1.82(4-13.6) 6.70^{* * *}, 21.26$ & $8.59 \pm 3.33(4-8.35) 2.51,38.75$ & $8.50 \pm 2.35(0.43)$ \\
\hline 7 & $\mathrm{PHT}(\mathrm{cm})$ & $175.79 \pm 38.94(85-256) 1842^{\star *}, 22.15$ & $172.20 \pm 39.46(74-281) 1,956.11^{* * *}, 22.92$ & $173.28 \pm 37.67(75-272) 1397.29^{* * *}, 21.74$ & $173.03 \pm 38.85(1575.74)$ \\
\hline 8 & $\mathrm{EHT}(\mathrm{cm})$ & $81.58 \pm 28.24^{\mathrm{ab}}(10-161) 1301^{* * *}, 34.61$ & $80.14 \pm 28.73^{\mathrm{b}}(25-171) 1,265.78^{\star * *}, 35.85$ & $83.89 \pm 27.07^{\mathrm{a}}(22.5-180) 1035.22^{* * *}, 32.27$ & $81.49 \pm 28.20\left(2999.93^{\star}\right)$ \\
\hline 9 & $\mathrm{StD}(\mathrm{mm})$ & $20.04 \pm 4.42(10-28) 11.79^{*}, 22.07$ & $19.42 \pm 4.24(10-48.5) 18.65^{\star * *}, 21.84$ & $19.49 \pm 3.91(10-28) 9.01^{* *}, 20.08$ & $19.52 \pm 4.17(47.11)$ \\
\hline 10 & SG (\%) & $79.09 \pm 14.75^{\mathrm{b}}(50-100) 206.90,18.65$ & $77.59 \pm 19.26^{b}(25-100) 1,179.35^{\star * *}, 24.81$ & $82.71 \pm 13.45^{\mathrm{a}}(50-100) 307.67^{\star * \star}, 16.26$ & $79.36 \pm 4.17\left(5560.32^{* \star \star}\right)$ \\
\hline 11 & $\mathrm{EL}(\mathrm{cm})$ & $16.47 \pm 2.84(10.5-23) 0.02^{* * *} 17.26$ & $16.12 \pm 3.5(7.5-28) 0.01^{* * *} 21.69$ & $16.51 \pm 3.42(7.5-26) 13^{* * *} 20.73$ & $16.29 \pm 3.4\left(536.24^{\star \star \star}\right)$ \\
\hline 12 & $\mathrm{ED}(\mathrm{mm})$ & $40.64 \pm 8.42^{\mathrm{ab}}(23-56) 8.21^{* \star *}, 20.72$ & $40.17^{\mathrm{b}} \pm 7.71(18.5-59.8) 3.15^{\star \star *}, 19.21$ & $41.45 \pm 7.46^{\mathrm{a}}(23-62.9) 2.24^{\star}, 18.00$ & $40.62 \pm 7.76\left(351.69^{\star \star}\right)$ \\
\hline 13 & $\mathrm{CD}(\mathrm{mm})$ & $25.06 \pm 5.19^{b}(2.7-35) 1.92,20.70$ & $26.66 \pm 6.39^{a}(12-50) 13.49^{* \star *}, 23.98$ & $27.13 \pm 5.71^{a}(11-49.1) 20.15^{\star \star \star}, 21.04$ & $26.58 \pm 6.07\left(450.04^{\star * *}\right)$ \\
\hline 14 & EN & $\left.1.01 \pm 0.04^{c}(1-1.3)\right) 6.51^{\star * \star}, 4.46$ & $1.02 \pm 0.05^{\mathrm{b}}(1-1.2) 12.50^{* * *}, 5.15$ & $1.06 \pm 0.17^{\mathrm{a}}(1-2) 8.95^{\star \star \star}, 15.92$ & $1.03 \pm 0.10\left(0.33^{\star * \star}\right)$ \\
\hline 15 & NRE & $11.66 \pm 2.67^{\mathrm{a}}(8-22) 103.21^{\star \star \star}, 22.94$ & $12.61^{\mathrm{b}} \pm 2.27(8-20) 69.33^{\star * *}, 17.99$ & $13.46 \pm 2.65^{\mathrm{b}}(8-22) 65.97^{\star}, 19.71$ & $12.74 \pm 2.52\left(355.10^{\star \star \star}\right)$ \\
\hline 16 & NKR & $32.69 \pm 6.33^{\mathrm{c}}(16-51) 865.73^{\star \star \star}, 19.38$ & $29.55 \pm 8.30^{b}(11-55) 647.61^{\star * *}, 28.08$ & $28.63 \pm 8.24^{\mathrm{a}}(13-55) 571.46^{\star \star \star}, 28.77$ & $29.70 \pm 8.13\left(1727.58^{\star \star \star}\right)$ \\
\hline 17 & HKW (kg) & $72.74 \pm 20.34^{\mathrm{a}}(42 .-147.) 13.11^{*}, 27.97$ & $67.64 \pm 21.54^{\mathrm{b}}(23.4-159.4) 23.30^{* * *}, 31.84$ & $66.43 \pm 19.97^{b}(30.5-128.9) 9.66^{\star * *}, 30.06$ & $67.98 \pm 20.99\left(4266.90^{\star \star *}\right)$ \\
\hline 18 & $\mathrm{KL}(\mathrm{mm})$ & $9.79 \pm 1.73^{\mathrm{a}}(6.5-12.8) 2.94^{\star \star}, 17.64$ & $9.54 \pm 1.65^{\mathrm{b}}(5.5-14.8) 3.48^{\star \star *}, 17.33$ & $9.29 \pm 1.69^{c}(6.0-15.0) 3.11^{\star * *} 18.20$ & $9.49 \pm 1.68\left(28.36^{\star * \star}\right)$ \\
\hline 19 & $\mathrm{KW}(\mathrm{mm})$ & $9.91 \pm 1.14^{\mathrm{a}}(7.5-12.5) 1.33^{* * *}, 11.54$ & $9.62 \pm 1.24^{b}(5-13.0) 1.55^{\star \star *}, 12.94$ & $9.19 \pm 1.31^{c}(4.9-13.2) 0.73^{* * *}, 14.20$ & $9.53 \pm 1.27\left(64.44^{\star \star \star}\right)$ \\
\hline 20 & $\mathrm{KT}(\mathrm{mm})$ & $4.89 \pm 0.76^{\mathrm{b}}(3.9-7.4) 0.00^{\star \star}, 15.56$ & $5.12 \pm 0.86^{\mathrm{a}}(5-13.0) 0.06^{\star \star \star}, 16.75$ & $5.16 \pm 0.78^{a}(3.5-8.4) 0.00^{* \star *}, 15.08$ & $5.10 \pm 0.83\left(8.29^{\star \star \star}\right)$ \\
\hline 21 & EWT $(\mathrm{kg})$ & $0.12 \pm 0.07^{\mathrm{b}}(0.03-0.3) 0.36^{\star \star}, 60.86$ & $0.14 \pm 0.13^{\mathrm{a}}(0.0-1.0) 114.98^{\star \star \star}, 91.16$ & $0.11 \pm 0.06^{\mathrm{b}}(0.0-0.3) 174.43^{\star * \star}, 57.15$ & $0.13 \pm 0.11\left(0.25^{\star * \star}\right)$ \\
\hline 22 & YLD (Mg ha $\left.{ }^{-1}\right)$ & $4.92 \pm 2.70^{\mathrm{a}}(1.89-11.6) 0.36,54.88$ & $4.5 \pm 2.74^{\mathrm{b}}(0.7-12.5) 0.26^{\star \star \star}, 61.15$ & $4.29 \pm 2.11^{\mathrm{b}}(0.7-9.5) 0.13^{\star}, 49.17$ & $4.48 \pm 2.56\left(41.20^{\star \star}\right)$ \\
\hline
\end{tabular}

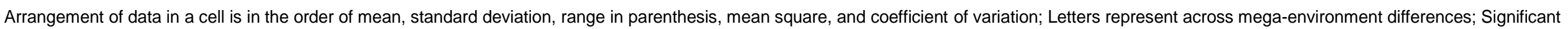
mean squares are represented as ${ }^{\star} P<0.05 ;{ }^{* *} P<0.01$, ${ }^{* * *} P<0.001$.

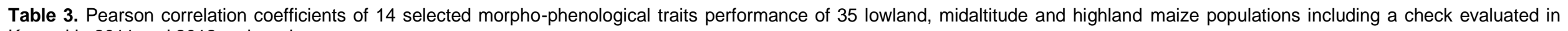
Kumasi in 2011 and 2012 major rainy seasons.

\begin{tabular}{|c|c|c|c|c|c|c|c|c|c|c|c|c|c|c|}
\hline & ASI & $\mathrm{TL}$ & ELL & ELW & PHT & EHT & StD & SG & EL & ED & HKW & $\mathrm{KL}$ & $\mathrm{KT}$ & YLD \\
\hline$A D$ & $0.32(0.05)$ & $0.21(0.21)$ & $0.25(0.14)$ & $0.05(0.77)$ & $0.36(0.03)$ & $0.46(0.00)$ & $0.40(0.01)$ & $0.06(0.70)$ & $0.35(0.03)$ & $-0.11(0.53)$ & $0.12(0.47)$ & $0.14(0.42)$ & $0.01(0.96)$ & $-0.14(0.40)$ \\
\hline ASI & & $0.15(0.38)$ & $0.17(0.32)$ & $-0.11(0.53)$ & $-0.11(0.52)$ & $-0.06(0.72)$ & $0.01(0.97)$ & $0.00(0.98)$ & $0.22(0.19)$ & $0.10(0.57)$ & $-0.02(0.90)$ & $-0.14(0.41)$ & $0.21(0.22)$ & $-0.26(0.13)$ \\
\hline $\mathrm{TL}$ & & & $0.58(0.00)$ & $0.49(0.00)$ & $0.42(0.01)$ & $0.33(0.05)$ & $0.32(0.05)$ & $0.26(0.11)$ & $0.56(0.00)$ & $0.39(0.02)$ & $0.45(0.00)$ & $0.35(0.03)$ & $0.17(0.31)$ & $0.33(0.05)$ \\
\hline ELL & & & & $0.57(0.00)$ & $0.48(0.00)$ & $0.50(0.00)$ & $0.51(0.00)$ & $0.45(0.01)$ & $0.67(<0.01)$ & $0.33(0.05)$ & $0.54(0.00)$ & $0.24(0.15)$ & $0.40(0.01)$ & $0.26(0.13)$ \\
\hline ELW & & & & & $0.46(0.00)$ & $0.49(0.00)$ & $0.56(0.00)$ & $0.43(0.01)$ & $0.61(<0.01)$ & $0.47(0.00)$ & $0.69(<0.01)$ & $0.56(0.00)$ & $0.46(0.00)$ & $0.60(0.00)$ \\
\hline
\end{tabular}


Table 3. Contd.

\begin{tabular}{|c|c|c|c|c|c|c|c|c|c|}
\hline PHT & $0.91(<0.01)$ & $0.66(<0.01)$ & $0.49(0.00)$ & $0.58(0.00)$ & $0.24(0.15)$ & $0.49(0.00)$ & $0.20(0.24)$ & $0.37(0.02)$ & $0.48(0.00)$ \\
\hline EHT & & $0.63(<0.01)$ & $0.53(0.00)$ & $0.52(0.00)$ & $0.08(0.62)$ & $0.42(0.01)$ & $0.18(0.27)$ & $0.37(0.03)$ & $0.41(0.01)$ \\
\hline StD & & & $0.42(0.01)$ & $0.80(<0.01)$ & $0.28(0.10)$ & $0.47(0.00)$ & $0.27(0.11)$ & $0.40(0.01)$ & $0.34(0.04)$ \\
\hline SG & & & & $0.40(0.01)$ & $0.28(0.09)$ & $0.39(0.02)$ & $0.05(0.78)$ & $0.51(0.00)$ & $0.38(0.02)$ \\
\hline EL & & & & & $0.47(0.00)$ & $0.58(0.00)$ & $0.28(0.09)$ & $0.49(0.00)$ & $0.30(0.07)$ \\
\hline ED & & & & & & $0.55(0.00)$ & $0.36(0.03)$ & $0.25(0.13)$ & $0.41(0.01)$ \\
\hline HKW & & & & & & & $0.60(<0.01)$ & $0.52(0.00)$ & $0.63(<0.01)$ \\
\hline KL & & & & & & & & $-0.12(0.47)$ & $0.56(0.00)$ \\
\hline KT & & & & & & & & & $0.17(0.30)$ \\
\hline
\end{tabular}

Figures in parenthesis are probability values.

Table 4. Genetic similarity among 36 highland, midaltitude, and lowland African landraces based on Pearson correlation coefficient on 22 morpho-phenological traits.

\begin{tabular}{llllllllllll}
\hline S/N & Acc & Min & Max & Average & SD & No. & Acc & Min & Max & Average & SD \\
\hline 1 & Obatanpa & 0.00 & 0.82 & 0.34 & 0.22 & 19 & TZm-1523 & 0.00 & 0.72 & 0.29 & 0.22 \\
2 & TZm-1084 & 0.00 & 0.58 & 0.26 & 0.19 & 20 & TZm-2 & 0.00 & 0.70 & 0.21 & 0.18 \\
3 & TZm-1095 & 0.00 & 0.58 & 0.24 & 0.14 & 21 & TZm-20 & 0.00 & 0.76 & 0.24 & 0.18 \\
4 & TZm-13 & 0.02 & 0.58 & 0.26 & 0.16 & 22 & TZm-242 & 0.01 & 0.58 & 0.23 & 0.17 \\
5 & TZm-1356 & 0.01 & 0.54 & 0.20 & 0.13 & 23 & TZm-251 & 0.02 & 0.80 & 0.31 & 0.18 \\
6 & TZm-1358 & 0.02 & 0.69 & 0.22 & 0.17 & 24 & TZm-270 & 0.01 & 0.62 & 0.24 & 0.17 \\
7 & TZm-1359 & 0.05 & 0.73 & 0.31 & 0.20 & 25 & TZm-273 & 0.01 & 0.67 & 0.27 & 0.17 \\
8 & TZm-1360 & 0.00 & 0.59 & 0.21 & 0.17 & 26 & TZm-275 & 0.02 & 0.62 & 0.28 & 0.17 \\
9 & TZm-1367 & 0.01 & 0.76 & 0.30 & 0.21 & 27 & TZm-301 & 0.04 & 0.62 & 0.30 & 0.17 \\
10 & TZm-1376 & 0.00 & 0.72 & 0.29 & 0.22 & 28 & TZm-33 & 0.01 & 0.62 & 0.22 & 0.14 \\
11 & TZm-14 & 0.01 & 0.58 & 0.22 & 0.17 & 29 & TZm-37 & 0.00 & 0.80 & 0.28 & 0.20 \\
12 & TZm-1413 & 0.01 & 0.82 & 0.34 & 0.20 & 30 & TZm-384 & 0.00 & 0.72 & 0.26 & 0.20 \\
13 & TZm-1424 & 0.01 & 0.71 & 0.21 & 0.19 & 31 & TZm-385 & 0.01 & 0.73 & 0.31 & 0.18 \\
14 & TZm-1434 & 0.00 & 0.71 & 0.28 & 0.19 & 32 & TZm-4 & 0.01 & 0.57 & 0.22 & 0.15 \\
15 & TZm-1437 & 0.00 & 0.58 & 0.20 & 0.15 & 33 & TZm-41 & 0.00 & 0.59 & 0.21 & 0.15 \\
16 & TZm-1514 & 0.00 & 0.58 & 0.26 & 0.15 & 34 & TZm-42 & 0.00 & 0.71 & 0.22 & 0.19 \\
17 & TZm-1516 & 0.01 & 0.59 & 0.24 & 0.16 & 35 & TZm-5 & 0.01 & 0.56 & 0.32 & 0.16 \\
18 & TZm-1521 & 0.00 & 0.80 & 0.32 & 0.20 & 36 & TZm-8 & 0.01 & 0.68 & 0.28 & 0.17 \\
\hline
\end{tabular}

of Midaltitude Tanzania. Average similarity coefficients of the accessions revealed that 16 genotypes were most distant, namely, 0.20 (TZm1356, TZm-1437), 0.21 (TZm-2, TZm-41, TZm-
1360, TZm-1424), 0.22 (TZm-4, TZm-14, TZm-33, $\mathrm{TZm}-42, \quad \mathrm{TZm}-1358, \quad 0.23$ (TZm-242), 0.24 


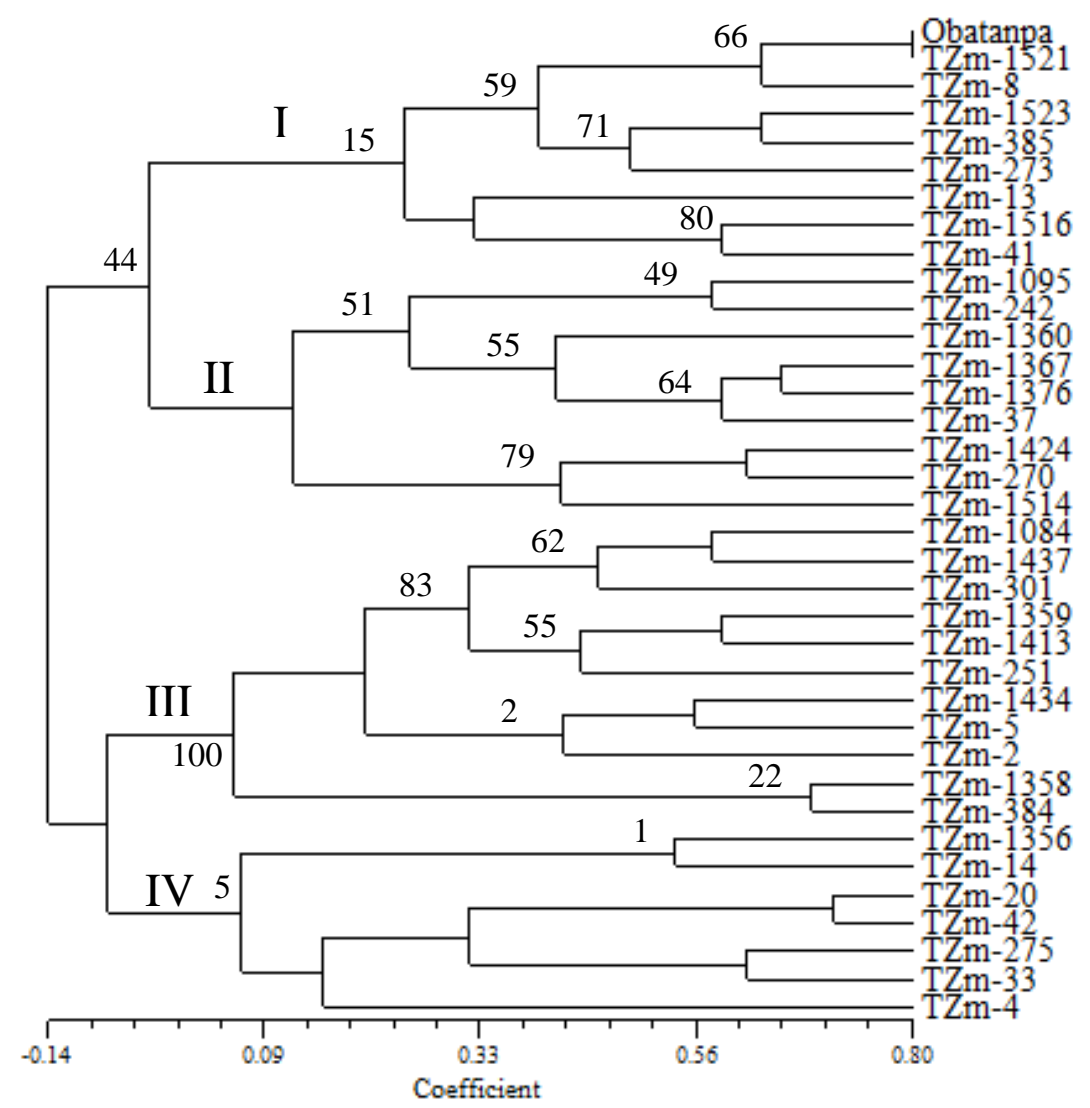

Figure 1. A UPGMA dendrogram of 22 quantitative agro- phenological traits based on Pearson correlation coefficients on 35 highland, midaltitude, and highland maize landraces and a check. Bootstrap values are shown at the nodes.

(TZm-20), TZm-270, TZm-1516, and TZm-1095. All distant accessions exhibited high yields exceeding 4.0 Mg ha ${ }^{-1}$ except TZm-2, TZm-20, TZm-42, and TZm-1516.

In all, 572 accession pairs (92\%) had similarity coefficients of less than 0.50 , while 49 were at least 0.50 . The preponderance of very low similarity coefficients is indicative of large genetic diversity among the accessions. As expected, accessions from different populations were more dissimilar than those of similar origins as was also corroborated by Beyene et al. (2006).

\section{Cluster analysis}

A UPGMA cluster analysis displayed four clusters (Figure 1) differentiated predominantly by geographic origin and confirmed seven accessions to be distant TZm-42, TZm20, TZm-5, TZm-2, TZm-270, TZm-41, and 'Obatanpa $\mathrm{GH}$ '. Cluster I had eight accessions, six of which originated from Midaltitude regions and two, including the check 'Obatanpa GH' from lowland regions. The genotypes exhibited early maturity, long ear leaf length, large ear and cob diameters, and long anthesis-silking interval. Cluster II, a mixed group with nine accessions, were mostly collected from all three mega environments. All highland accessions except one belonged to this cluster most of which exhibited shortest anthesis-silking interval, large tassels, large ear leaf widths, largest stalk diameter, tall plants, and highest mean grain yield of 5.20 $\mathrm{Mg} \mathrm{ha}^{-1}$, which exceeded the overall mean yield by 0.72 $\mathrm{Mg} \mathrm{ha}{ }^{-1}$. Five members of cluster II were distant TZm1095, TZm-242, TZm-1360, TZm-1424, and TZm-270. Cluster III had 11 accessions mostly of lowland and few Midaltitude genotypes with intermediate maturity, high stay green and intermediate grain yield exceeding mean grain yield by $0.12 \mathrm{Mg} \mathrm{ha}^{-1}$. Cluster IV was predominated by seven members of Midaltitude origin which were late maturing, short plants with small tassels, small ear leaf width, and least mean grain yield of $0.14 \mathrm{Mg} \mathrm{ha}^{-1}$ below the overall average yield (Table 5).

\section{Principal components analysis}

Principal components analysis delimited 21 important discriminatory descriptors. The first four principal 
Table 5. Overall mean, cluster means, and standard deviation of the 35 highland, midaltitude and lowland African maize landraces and a check evaluated in Ghana by morphological trait measurement.

\begin{tabular}{|c|c|c|c|c|c|}
\hline Trait & Overall & Cluster I & Cluster II & Cluster III & Cluster IV \\
\hline$A D$ & $58.60 \pm 5.67$ & $54.44 \pm 5.19(-4.16)$ & $59.19 \pm 4.54(0.58)$ & $58.78 \pm 5.44(0.18)$ & $59.88 \pm 4.68(1.28)$ \\
\hline SD & $62.68 \pm 6.10$ & $58.09 \pm 5.32(-4.59)$ & $62.74 \pm 4.95(0.06)$ & $62.85 \pm 5.82(0.17)$ & $64.58 \pm 5.03(1.89)$ \\
\hline ASI & $4.08 \pm 1.66$ & $3.65 \pm 1.31(-0.43)$ & $3.56 \pm 1.41(-0.53)$ & $4.07 \pm 1.66()-0.01$ & $4.70 \pm 1.71(0.62)$ \\
\hline TL & $47.14 \pm 7.34$ & $47.23 \pm 7.52(0.09)$ & $48.27 \pm 7.48(1.13)$ & $47.23 \pm 7.29(0.08)$ & $45.25 \pm 6.06(-1.90)$ \\
\hline ELL & $79.16 \pm 16.80$ & $80.18 \pm 16.54(1.02)$ & $78.52 \pm 18.35(-0.64)$ & $79.37 \pm 17.09(0.21)$ & $73.16 \pm 16.99(-6.00)$ \\
\hline ELW & $8.50 \pm 2.35$ & $8.69 \pm 3.55(0.19)$ & $9.00 \pm 1.65(0.50)$ & $8.50 \pm 1.63(0.00)$ & $7.70 \pm 1.72(-0.80)$ \\
\hline PLH & $173.03 \pm 38.85$ & $171.13 \pm 37.77(-1.90)$ & $180.47 \pm 40.95(7.44)$ & $176.47 \pm 37.75(3.44)$ & $162.76 \pm 35.58(-10.26)$ \\
\hline EHT & $81.49 \pm 28.20$ & $76.58 \pm 25.85(-4.90)$ & $85.93 \pm 27.90(4.45)$ & $84.10 \pm 28.06(2.62)$ & $72.98 \pm 27.90(-8.51)$ \\
\hline StD & $19.52 \pm 4.17$ & $19.45 \pm 4.18(-0.08)$ & $20.17 \pm 3.91(0.65)$ & $19.83 \pm 4.06(0.31)$ & $18.22 \pm 3.91(-1.31)$ \\
\hline SG & $79.36 \pm 17.21$ & $79.62 \pm 14.18(0.26)$ & $79.56 \pm 15.10(0.20)$ & $81.29 \pm 15.16(1.93)$ & $74.34 \pm 22.57(-5.02)$ \\
\hline EL & $16.29 \pm 3.4$ & $16.83 \pm 3.23(0.54)$ & $16.03 \pm 3.25(-0.26)$ & $16.26 \pm 3.59(-0.03)$ & $15.89 \pm 3.43(-0.4)$ \\
\hline EP & $0.46 \pm 0.09$ & $0.44 \pm 0.08(-0.02)$ & $0.47 \pm 0.09(0.01)$ & $0.47 \pm 0.09(0.01)$ & $0.44 \pm 0.10(-0.03)$ \\
\hline ED & $40.62 \pm 7.76$ & $42.11 \pm 7.03(1.49)$ & $40.94 \pm 7.81(0.31)$ & $40.80 \pm 7.86(0.18)$ & $41.04 \pm 6.86(0.42)$ \\
\hline CD & $26.58 \pm 6.07$ & $30.23 \pm 6.99(3.65)$ & $24.51 \pm 5.10(-2.07)$ & $26.39 \pm 5.57(-0.19)$ & $25.80 \pm 4.74(-0.78)$ \\
\hline NRE & $12.74 \pm 2.52$ & $13.26 \pm 1.99(0.52)$ & $11.39 \pm 2.41(-1.35)$ & $12.67 \pm 2.54(-0.07)$ & $12.28 \pm 2.31(-0.46)$ \\
\hline NKR & $29.70 \pm 8.13$ & $28.01 \pm 8.56(-1.70)$ & $32.64 \pm 6.59(2.93)$ & $29.61 \pm .38(-0.09)$ & $29.38 \pm 7.94(-0.33)$ \\
\hline HKW & $67.98 \pm 20.99$ & $67.45 \pm 19.02(-0.53)$ & $76.49 \pm 22.03(8.51)$ & $68.87 \pm 19.91(0.89)$ & $64.15 \pm 17.35(-3.83)$ \\
\hline EN & $1.03 \pm 0.10$ & $1.02 \pm 0.05(-0.01)$ & $1.01 \pm 0.04(-0.02)$ & $1.03 \pm 0.07(-0.01)$ & $1.03 \pm 0.06(0.00)$ \\
\hline $\mathrm{KL}$ & $9.49 \pm 1.68$ & $9.33 \pm 1.71(-0.16)$ & $10.30 \pm 1.54(0.81)$ & $9.47 \pm 1.64()-0.02$ & $9.44 \pm 1.40(-0.06)$ \\
\hline KW & $9.53 \pm 1.27$ & $9.64 \pm 0.98(0.11)$ & $10.17 \pm 1.04(0.64)$ & $9.59 \pm 1.18(0.06)$ & $9.73 \pm 0.91(0.20)$ \\
\hline KT & $5.10 \pm 0.83$ & $5.24 \pm 0.77(0.14)$ & $5.07 \pm 0.72(-0.03)$ & $5.17 \pm 0.80(0.07)$ & $4.83 \pm 0.74()-0.28$ \\
\hline EWT & $0.13 \pm 0.11$ & $0.12 \pm 0.08(-0.02)$ & $0.13 \pm 0.07(-0.01)$ & $0.12 \pm 0.09(-0.01)$ & $0.18 \pm 0.16(0.05)$ \\
\hline GWT & $0.83 \pm 0.47$ & $0.82 \pm 0.45(-0.01)$ & $0.96 \pm 0.50(0.13)$ & $0.85 \pm 0.46(0.02)$ & $0.80 \pm 0.49(-0.03)$ \\
\hline YLD & $4.48 \pm 2.56$ & $4.44 \pm 2.41(-0.04)$ & $5.20 \pm 2.73(0.72)$ & $4.60 \pm 2.50(0.12)$ & $4.34 \pm 2.65(-0.14)$ \\
\hline
\end{tabular}

${ }^{1}$ Numbers in parenthesis represent differences between the cluster and overall mean; the legends for traits and their units are as given in Table 2.

components with eigenvalues exceeding 1.0 explained $86.67 \%$ of the total phenotypic variance (Table 6 ). In the first PC which accounted for $39.97 \%$, the variance was attributed to the weighted sum of tassel length, ear leaf length, ear leaf width, plant height, ear position, stalk diameter, stay green, ear length, ear diameter, hundred kernel weight, kernel length, and grain yield. This indicates a direct relationship between plant architectural traits, stay green, grain yield and yield components. Accessions having this combination of positively loading traits represent positive associations that are likely to share common alleles. Crossing within this gene pool would accumulate alleles for large values of tassel size, ear leaf length and width, stay green, yield components and grain yield.

The PC2 explained $18.70 \%$ of the total variance attributed to the weighted sum of number of kernels per row, kernel length, kernel width and ear weight, and a weighted difference of cob diameter, number of rows per ear and kernel thickness. A variance contribution in PC3 of $16.74 \%$ was attributed to weighted sum of anthesis and silking dates and anthesis-silking interval but a weighted difference in ear diameter and grain yield, indicating an inverse relationship between maturity date, anthesis-silking interval and grain yield. In other words, the late maturing genotypes with long anthesis-silking interval exhibited low grain yield. Being landraces, it was not unexpected to find genotypes exhibiting undesirable traits such as large anthesis-silking interval and low grain yield. The contribution of PC4 of $11.06 \%$ of the total variance was attributed to the weighted difference between anthesis-silking interval and ear height (Table $6)$.

A trait biplot of PC1 and PC2 (Figure 2A) accounted for a cumulative variance of $58.87 \%$ and identified four major correlation groupings, namely, a group based on earliness, plant architectural traits and grain yield; a second group based on ear-related traits; a third group based on kernel characteristics; and lastly, an uncorrelated group of traits comprising number of rows per ear, anthesis-silking interval, cob diameter and number of kernels per row. The tight angles between anthesis and silking dates, kernel width and kernel length, stay green and kernel thickness, ear leaf length and ear leaf width, plant height and grain yield signified direct association among these traits. In contrast, anthesis-silking interval, cob diameter, number of rows per ear, and number of ears per plant were uncorrelated. 
Table 6. Eigenvalues, eigenvectors, and cumulative percentage of variation explained by the first four principal components (PC) on 22 morphological traits in 35 maize landraces from lowland, midaltitude, and highland region of Africa including a check.

\begin{tabular}{lcccc}
\hline Variable & PC1 & PC2 & PC3 & PC4 \\
\hline AD & 0.37 & 0.42 & $\mathbf{0 . 7 6}$ & 0.10 \\
SD & 0.35 & 0.36 & $\mathbf{0 . 7 9}$ & 0.19 \\
ASI & 0.04 & -0.15 & $\mathbf{0 . 5 0}$ & $\mathbf{0 . 5 8}$ \\
TL & $\mathbf{0 . 6 1}$ & 0.05 & -0.06 & 0.32 \\
ELL & $\mathbf{0 . 7 3}$ & -0.11 & 0.10 & 0.12 \\
ELW & $\mathbf{0 . 8 0}$ & -0.11 & -0.29 & 0.01 \\
PLHT & $\mathbf{0 . 8 0}$ & 0.05 & 0.15 & -0.31 \\
EHT & $\mathbf{0 . 7 8}$ & 0.01 & 0.32 & -0.44 \\
StD & $\mathbf{0 . 7 8}$ & 0.04 & 0.17 & -0.02 \\
SG & $\mathbf{0 . 6 1}$ & -0.41 & -0.01 & -0.29 \\
EL & $\mathbf{0 . 8 2}$ & -0.05 & 0.13 & 0.27 \\
ED & $\mathbf{0 . 5 0}$ & -0.12 & -0.45 & 0.50 \\
CD & 0.30 & $-\mathbf{0 . 5 4}$ & -0.21 & 0.36 \\
EN & -0.26 & -0.35 & -0.05 & -0.15 \\
NRE & 0.06 & $\mathbf{- 0 . 7 5}$ & 0.08 & 0.13 \\
NKR & 0.04 & $\mathbf{0 . 6 5}$ & -0.32 & -0.32 \\
HKWT & $\mathbf{0 . 7 9}$ & 0.15 & -0.34 & 0.13 \\
KL & 0.48 & $\mathbf{0 . 5 6}$ & -0.42 & 0.09 \\
KW & 0.45 & $\mathbf{0 . 5 7}$ & -0.40 & 0.23 \\
KT & $\mathbf{0 . 5 4}$ & $\mathbf{- 0 . 5 5}$ & 0.08 & 0.08 \\
EWT & -0.20 & $\mathbf{0 . 7 1}$ & 0.21 & 0.34 \\
YLD & $\mathbf{0 . 6 0}$ & 0.08 & -0.54 & -0.25 \\
Eigenvalues & 6.10 & 2.97 & 2.60 & 1.76 \\
Individual percentage & 39.97 & 18.90 & 16.74 & 11.06 \\
Cumulative variance $(\%)$ & 39.97 & 58.87 & 75.60 & 86.67 \\
\hline
\end{tabular}

Strong associations were exhibited between stay green and kernel thickness. Plants with longer stay green also had large kernel thickness. In the accession biplot (Figure 2B), three uncorrelated groups and eight accessions that were separated from the core groups were identified, confirming that TZm-270, TZm-2, TZm-5, TZm-251, TZm42, TZm-385, TZm-41 and TZm-1358 were distant.

Hybridization within clusters I and III should be favorable in accumulating alleles for early maturity, low to moderate anthesis-silking interval, stay green, and high grain yield. On the contrary, accessions of cluster IV had predominance of small plant architectural traits and low yield, low values of stay green and longest anthesissilking interval.

In the context of maize improvement, a breeding strategy that would exploit the existing variation within and between the clusters and accumulate desirable characteristics is to create a cross-pollinated population with a high frequency of high grain yield, early maturing, low anthesis-silking interval and high stay green genotypes and improve this group via recurrent selection to obtain highly heterozygous populations from which superior heterozygous genotypes may be selected for inbred line development.

\section{DISCUSSION}

The African maize landraces constitute an important class of genotypes characterized by wide diversity in phenology, plant growth, grain yield, and leaf photosynthesis most of which connote a diversity of farmer preferences and adaptive characteristics to a wide range of environments in which they have evolved. This diversity awaits to be exploited for maize improvement in an efficient manner. Noteworthy was the identification of some genotypes that outperformed the improved cultivar in grain yield, leaf photosynthesis and phenology.

The simultaneous large within and large between population variations is the likely result of large rate of gene flow between midaltitude and lowland populations but somehow restricted between the geographically discrete highland genotypes. The variation highlights a naturally conserving potential which has been shaped by evolutionary factors including a large effective population size with a large proportion of gene flow which does not 

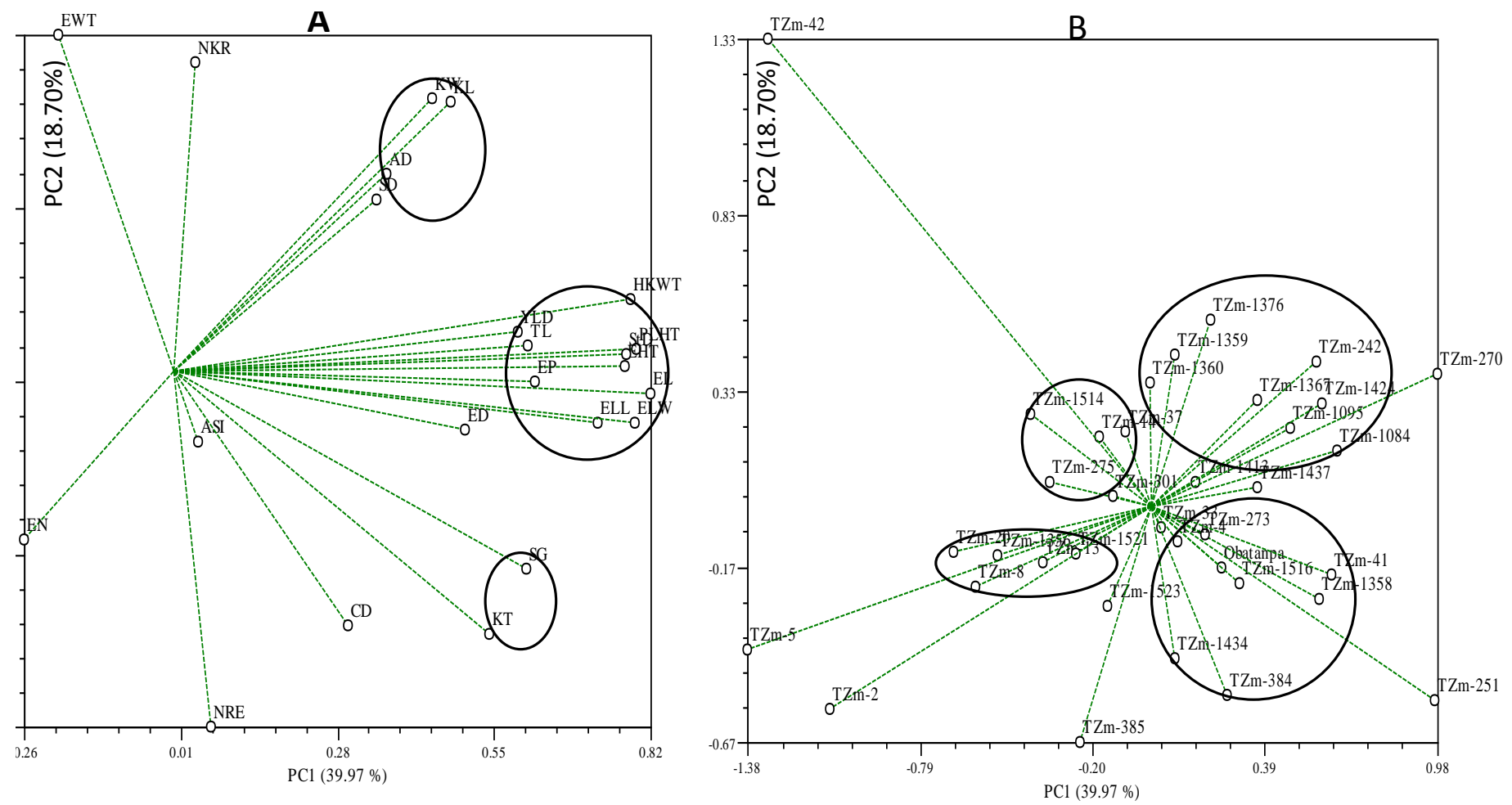

Figure 2. Plots of PC1 against PC2 for (A) traits and (B) 35 accessions and "Obatanpa GH", the check. The labels are as defined in Table 2 footnote.

disrupt patterns of local adaptation of the taxon. Marshall (1977) reported that the landraces possess both within and between variations. A PCR-based study on genetic diversity, as well as estimation of $F_{\mathrm{ST}}, F_{\mathrm{SC}}$, and $G_{\mathrm{ST}}$ are needed to confirm the wide within and among diversity in the African maize landraces.

The larger variability in the midaltitude and lowland accessions was not surprising considering that the major entry of maize to Africa was through the west coast rather than the east or north. In fact, besides midaltitude Tanzania and Zambia, many of the lowland and midaltitude accessions in current study originated from West African countries such as, Togo, Congo, Guinea, Equatorial Guinea, and Chad. The fewer representations of the highland genotypes may have influenced the low variation observed.

The overall mean anthesis dates of $58.62 \pm 5.67$ days and silking date of $62.68 \pm 6.10$ days were similar to the $61.5 \pm 0.2$ days and silking date of $62.7 \pm 0.7$ days (Salami et al., 2007), but shorter than anthesis and silking dates of $65.1 \pm 3.2$ and $71.5 \pm 3.0$ days (Beyene et al., 2006), $83.0 \pm 0.5$ days and $85.9 \pm 0.55$ days (Azad et al., 2012), $84.3 \pm 1.7$ and $86.6 \pm 12.0$ days of 498 maize accessions of Asia, Latin America and U.S.A. (Weiwei et al., 2012). Early maturing genotypes that may be useful to breeding programs in marginal regions in tropical Africa were TZm8, TZm-2, TZm-1521, and TZm-385. The short anthesissilking interval of TZm-1376 (2.3 days) would benefit breeding for drought tolerance through escape and avoidance. The positive association of ear leaf width with grain yield was noteworthy and would be relevant in selection based on correlated response.

The genotypes of current study had plant heights similar to those of Ethiopia (161.0 to $288.0 \mathrm{~cm}$ with a mean of $217.8 \pm 14.4 \mathrm{~cm}$ ) (Beyene et al., 2006) and Italian landraces ( 110.0 to $215.0 \mathrm{~cm}$ with a mean of $166.0 \pm 27.4$ (Hartings et al., 2008). Candidate genotypes having mean grain yield in excess of $5.0 \mathrm{Mg} \mathrm{ha}^{-1}$ including TZm33, TZm-41, TZm-14, TZm-242, TZm-37, TZm-1360, $\mathrm{TZm}-1376, \mathrm{TZm}-1367, \mathrm{TZm}-4$, and TZm-270 would benefit breeding for high grain yield.

Genetic distance within the accessions confirmed a large variability which was further validated by cluster analysis and principal components analyses. TZm-42, TZm-2 and TZm-5 exhibited least values of grain yield and yield-related traits of hundred kernel weight, stay green, and stalk diameter, ear leaf width and tassel length. In contrast, TZm-270 demonstrated largest values of these traits, unequalled with all other accessions and would be worthy to incorporate into breeding programs.

Existence of both within and among population variation presents a special opportunity for in situ conservation which in Africa is a common traditional farming practice to check genetic erosion and ensure maintenance of the evolutionary processes in the taxon while adopting modern agricultural technology (Brush, 
1995). The corollary of this conservation is environmental resilience and creation of novel genotypes (Altieri and Merrik; 1987; Friis-Hansen, 1994). Additionally, the partitioning of the populations into clusters suggests that benefits would be accrued from intercrossing between clusters to exploit heterosis for grain yield, earliness, and the desirable low values of anthesis-silking interval which indicate less sensitivity to drought stress. A within cluster crossing involving a large number of ears should preserve the rare alleles in the collection (Crossa, 1989).

The combined high yield and early-maturity traits present novel genes at these loci and may be beneficial for broadening the genetic base of elite gene pools.

\section{Conclusion}

The findings of this work suggest that the African maize landraces are rich in genetic diversity which increases from highland to lowland and to midaltitude genotypes. The large difference between population differentiation and the rich diversity suggest a historic formation from a large effective population size. At the same time, the large within population variability indicates a gene flow among the populations which is consistent with an ex situ conservation of maize. The morphological variability can provide basis for creating core subsets especially from the two highly populated clusters. Identification of the large variabilities among the traits bring to fore the richness in alleles and the urgency to conserve and incorporate the landraces into regional breeding programs to widen the genetic base of the genepool. These genotypes have adapted to the environmental conditions of Africa and are expected to contribute alleles for both trait improvement and to withstand environmental stress factors in time and space.

In terms of world average maize yield of $5.15 \mathrm{Mg} \mathrm{ha}^{-1}$, the important genotypes and their respective grain yields were TZm-14 (5.2 Mg ha $\left.{ }^{-1}\right), \mathrm{TZm}-41\left(5.2 \mathrm{Mg} \mathrm{ha}^{-1}\right), \mathrm{TZm}-$ 242 (5.3 Mg ha $\left.{ }^{-1}\right), T Z m-37$ (5.4 Mg ha $\left.{ }^{-1}\right), T Z m-1360$ (5.6 $\left.\mathrm{Mg} \mathrm{ha}^{-1}\right), \mathrm{TZm}-1376$ (5.6 Mg ha-1), TZm-1367 (6 Mg ha $\left.{ }^{-1}\right)$, TZm-4 (6.0 Mg ha $\left.{ }^{-1}\right)$, and TZm-270 (6.2 Mg ha $\left.{ }^{-1}\right)$, all of which were midaltitude and highland accessions.

A unique combination of early-maturing and highyielding traits in TZm-14 and TZm-33 TZm-37, TZm1367, and TZm-1376 may offer opportunity to breed for combined earliness and yield, which usually present a trade-off. Unusually large number of kernels per row (48 to 54) identified in nine accessions, namely, TZm-1521 and TZm-41 (48), TZm-14 (49), TZm-1356, TZm-301 and TZm-1360 (50), TZm-1359 (52), TZm-1084 and TZm-273 (54) may be considered for further evaluation for grain yield improvement.

\section{Conflicts of Interests}

The authors declare that they have no conflict of interest.

\section{ACKNOWLEDGEMENTS}

The authors express their gratitude to the Kwame Nkrumah University of Science and Technology for providing grant for the research.

\section{REFERENCES}

Altieri MA, Merrik LC (1987). In situ conservation of crop genetic resources through maintenance of traditional farming systems. Econ. Bot. 41:86-89.

Amanor KS (2013). Dynamics of maize seed production systems in the Brong Ahafo Region of Ghana; Agricultural modernization, farmer adaptive experimentation and domestic food markets. Future Agricultures, Working Paper 061. Pp. 1-20.

Azad MAK, Biswas BK, Alam N, Alam SS (2012). Genetic diversity in maize (Zea mays L.) inbred lines. A Scientific Journal of Krishi Foundation. Agriculturists 10:64-70.

Bellon MR (2004). Conceptualizing interventions to support on-farm genetic resource conservation. World Dev. 32:159-172.

Bellon MR, van Etten J (2013). Climate Change and On-Farm Conservation of Crop Landraces in Centres of Diversity. In Plant Genetic Resources and Climate Change. Jackson M, Ford-Lloyd, B. and Parry ML (Eds.). CABI Publishing, New York.

Beyene Y, Botha AM, Myburg AA (2006). Genetic diversity among traditional Ethiopian highland maize accessions assessed by simple sequence repeat (SSR) markers. Genet. Resour. Crop Evol. 53:1579-1588.

Botha AM, Venter E, (2000). Molecular marker technology linked to pest and pathogen resistance in wheat breeding. South Afr. J. Sci. 96:233240.

Brush SB (1995). In situ conservation of landraces in center of crop diversity. Crop Sci. 35:346-354.

Cairns JE, Hellin J, Sonder K, Araus JL, MacRobert JF, Thierfelder C, Prasanna M (2013). Adapting maize production to climate change in Sub-Saharan Africa. Food Secur. 5:345-360.

Carvalho VP, Ruas PM, Ruas CF, Ferreira JM, Moreira MP (2002). Assessment of genetic diversity in maize (Zea mays L.) landraces using Inter Simple Sequence Repeat (ISSR) markers. Crop Breed. Appl. Biotechnol. 2:557-568.

Crossa J (1989). Methodologies for estimating the sample size required for genetic conservation outbreeding crops. Theor. Appl. Genet. 77:153-161.

Enoki H, Sato H, Koinuma K (2002) SSR analysis of genetic diversity among maize inbred lines adapted to cold regions of Japan. Theor. Appl. Genet. 104:1270-1277.

FAO (2006). Global Perspective Studies Unit, Food and Agriculture Organization of the United Nations, Rome. World agriculture: towards 2030/2050, Interim report.

FAOSTAT (2013). Crop production Statistics. Food and Agriculture Organization, Rome. www.faostat.fao.org. Accessed up to September 01, 2013.

Felsenstein $\mathrm{J}$ (1985). Confidence limits on phylogenies: an approach using the bootstrap. Evolution 39:783-791.

Fischer T, Byerlee D, Edmeades G (2014). Crop yields and global food security. Will yield increase continue to feed the world? Australian Center for International Agricultural Research. ACIAR Monograph No. 158. Grains Research Development Corporation.

Friis-Hansen E (1994). Conceptualizing in situ conservation of landraces. In. A.F. Krattiger et al. (ed.) Widening perspectives on biodiversity. IUCN, Gland, Switzerland and International Academy of the Environment, Geneva. pp. 263-276.

Gibbon D, Dixon J, Flores D (2007). Beyond drought tolerant maize: study of additional priorities in maize. Report to Generation Challenge Program. Impacts, Targeting and Assessment Unit. Centro Internacional de Mejoramiento de Maiz y Trigo, Mexico D.F., 42p.

Goodman MM, Stuber CW (1983). Races of maize. VI. Isozyme variation among races of maize in Bolivia. Maydica 28:169-187.

Hammer $\varnothing$, Harper DAT, Ryan PD (2001). PAST: Paleontological Statistics Software Package for Education and Data Analysis. 
Palaeontol. Electronica 4:9.

Hartings $H$, Berardo N, Mazzinelli GF, Valoti P, Verderio A, Motto M (2008). Assessment of genetic diversity and relationships among maize (Zea mays L.) Italian landraces by morphological traits and AFLP profiling. Theor. Appl. Genet. 117:831-842.

IBPGRI and CIMMYT (1991).International Board for Plant Genetic Resources (IBPGR); International Maize and Wheat Improvement Center (CIMMYT).

IPCC-Intergovernmental Panel on Climate Change (2007). Climate Change 2007: The Physical Science Basis. Contribution of Working Group I to the Fourth Assessment Report of the Intergovernmental Panel on Climate Change. Solomon S, Qin D, Manning M, Chen Z, Marquis M, and Avery KB, et al. (Eds). Cambridge University Press: Cambridge, UK.

James GG, Joanne AL, Kendall RL, Margaret ES, Stephen K (2002). SSR variation in important U.S. maize inbred lines. Crop Sci. 42:951957.

Kahler AL, Hallauer AR, Gardner CO (1983). Allozyme polymorphisms within and among open-pollinated and adapted exotic populations of maize. Theor. Appl. Genet. 72:592-601.

Legesse BW, Myburg AA, Pixley KV, Botha AM (2007). Genetic diversity of African inbred lines revealed by SSR markers. Hereditas 144:10-17.

Magorokosho C (2006). Genetic diversity and performance of maize varieties from Zimbabwe, Zambia and Malawi. PhD Thesis, Texas A\&M University, U.S.A.

Marshall DR (1977). The advantages and hazards of genetic homogeneity. Ann. N.Y. Acad. Sci. 287:1-20.

Mercer KL, Perales HR (2010). Evolutionary response of landraces to climate change in centers of crop diversity. Evol. Appl. 3:480-493.

Mercer KL, Perales HR, Wainwright JD (2012). Climate change and the transgenic adaptation strategy: Smallholder livelihoods, climate justice, and maize landraces in Mexico. Glob. Environ. Change 22:495-504

Obeng-Antwi K (2007). Genetic diversity in Maize (Zea mays L.) landraces in Ghana. PhD Thesis. University of Reading, United Kingdom.

Obeng-Antwi K, Craufurd PQ, Menkir A, Ellis RH, Sallah PYK (2012). Phenotypic diversity in maize landraces in Ghana. Int. J. Sci. Adv. Technol. 2:2221-8386.

Okumus A (2007). Genetic variation and relationship between Turkish flint maize landraces by RAPD markers. Am. J. Agric. Biol. Sci. 2:4953.
Oppong A, Bedoya CA, Ewool MA, Asante MD, Thompson RN, AduDapaah H, Lamptey JN L, Ofori K, Offei SK, Warburton ML (2014). Bulk genetic characterization of Ghanaian maize landraces using microsatellite markers. Maydica 59:1-8.

Rebourg C, Gouesnard B, Charcosset A (2001). Large scale molecular analysis of traditional European maize populations. Relationships with morphological variation. Heredity 86:574-587.

Rohlf FJ (2009). NTSYSpc: Numerical Taxonomy System. Version 2.21c. Exeter Software: Setauket, New York.

Salami AE, Adegoke SAO, Adegbite OA (2007). Genetic variability among maize cultivars grown in Ekiti-State, Nigeria. Middle East $\mathrm{J}$. Sci. Res. 2:9-13.

Sanou J, Gouesnard B, Charrier A (1997). Isozyme variability in West African maize cultivars (Zea mays L.). Maydica 42:1-11.

SAS Institute (2011). Statistical Analysis System. SAS Version 9.3.1 SAS Institute, Cary, North Carolina

Smith JSC (1986). Genetic diversity within the Corn Belt dent racial complex of maize (Zea mays L.). Maydica 31:349-367.

Smith OS, Smith JSC (1992). Measurement of genetic diversity among hybrids: A comparison of isozymic, RFLP, pedigree, and heterosis data. Maydica 37:53-60.

Warburton ML, Ribaut JM, Franco J, Crossa J, Dubreuil P, Betrán FJ (2005). Genetic characterization of 218 elite CIMMYT maize inbred lines using RFLP markers. Euphytica 142:97-106.

Warburton ML, Xia X, Crossa J, Franco J, Melchinger AE, Frisch M, Bohn M, Hoisington D (2002). Genetic characterization of CIMMYT inbred maize lines and open pollinated populations using large scale fingerprinting methods. Crop Sci. 42:1832-1840.

Weiwei W, Franco J, Chavez-Tovar V, Jianbing H, Yan J, Taba S (2012). Genetic characterization of a core set of a tropical maize race Tuxpeno for further use in maize improvement. PLoS One 7:e32626.

Xia XC, Reif JC, Melchinger AE, Frisch M, Hoisington DA, Beck D, Pixley K, Warburton ML (2005). Genetic diversity among CIMMYT maize inbred lines investigated with SSR markers: II. Subtropical, tropical mid-altitude, and highland maize inbred lines and their relationships with elite U.S. and European maize. Crop Sci. 45:25732582.

Yuan LX, Fu JH, Warburton ML, Li XH, Zhang SH, Khairallah M, Liu X, Peng Z, Li L (2000). Comparison of genetic diversity among maize inbred lines based on RFLPs, SSRs, AFLPs, and RAPDs. Acta Genetica Sinica 27:725-733. 